\title{
Norane Pontos de corte da circunferência da cintura e da razão cintura/estatura para excesso de peso: estudo transversal com idosos de sete cidades brasileiras, 2008-2009*
}

doi: $10.5123 /$ S1679-49742020000400027

\author{
Waist circumference, waist-to-height ratio and overweight cut-off points: a cross-sectional \\ study with elderly people in seven Brazilian cities, 2008-2009
}
Circunferencia de la cintura, razón cintura/estatura y puntos de corte para exceso de peso: estudio transversal con adultos mayores de siete ciudades brasileñas, 2008-2009

\author{
Daniela de Assumpção' - (D orcid.org/0000-0003-1813-996X \\ Rosemeire de Olanda Ferraz' - D orcid.org/0000-0001-5796-946X \\ Flávia Silva Arbex Borim ${ }^{1}$ - (1) orcid.org/0000-0001-7316-1145 \\ Anita Liberalesso Neri' 1 - 10 orcid.org/0000-0002-6833-7668 \\ Priscila Maria Stolses Bergamo Francisco' - @ orcid.org/0000-0001-7361-9961 \\ 'Universidade Estadual de Campinas, Faculdade de Ciências Médicas, Campinas, SP, Brasil
}

\section{Resumo}

Objetivo: identificar pontos de corte para circunferência da cintura (CC) e razão cintura/estatura (RCE) com melhor sensibilidade, especificidade e acurácia para discriminar idosos com excesso de peso por sexo, utilizando-se três critérios de índice de massa corporal como referência antropométrica. Métodos: estudo transversal que analisou medidas aferidas de peso, estatura e CC de 3.477 idosos (2008-2009); utilizaram-se gráficos de curvas receiver operating characteristic (ROC) para definir os pontos de corte. Resultados: os pontos de corte para CC foram de $86,5 \mathrm{~cm}, 88,7 \mathrm{~cm}$ e $91,5 \mathrm{~cm}$ nas mulheres, e de 93,0cm, 96,0cm e 97,5cm nos homens, pelos critérios da Organização Mundial da Saúde (OMS), Nutrition Screening Initiative (NSI) e Organização Pan-Americana da Saúde (OPAS), respectivamente; para a RCE, corresponderam a 0,57, 0,58, 0,59 no sexo feminino, e 0,56, 0,58 e 0,59 no masculino; maiores valores absolutos de acurácia global, OPAS (homens) e OMS (mulheres). Conclusão: os pontos de corte de CC e RCE foram similares, independentemente do critério de IMC adotado.

Palavras-chave: Idosos; Circunferência da Cintura; Índice de Massa Corporal; Razão Cintura-Estatura; Inquéritos Epidemiológicos; Estudos Transversais.

\footnotetext{
* A pesquisa de que se originou este artigo contou com financiamento do Conselho Nacional de Desenvolvimento Científico e Tecnológico (CNPq, Processo no 55.5082/2006-7), da Coordenação de Aperfeiçoamento de Pessoal de Nível Superior (Capes, Processo nº 02-P-4532/2017) e da Fundação de Amparo à Pesquisa do Estado de São Paulo (Fapesp, Processo nº 2017/23245-0).
}

Endereço para correspondência:

Daniela de Assumpção - Rua Tessália Vieira de Camargo, no 126, Cidade Universitária Zeferino Vaz, Campinas, SP, Brasil. CEP: $13083-887$

E-mail:danideassumpcao@gmail.com 


\section{Introdução}

0 envelhecimento está associado a mudanças na composição corporal, reveladas pela redução da massa magra, aumento da massa gorda e redistribuição destes tecidos no corpo, com maior acúmulo de gordura intra-abdominal e intramuscular. ${ }^{1,2}$ Dados do Framingham Heart Study (2002-2005) mostram que tanto o tecido adiposo subcutâneo quanto o intra-abdominal/visceral estão correlacionados com fatores de risco cardiometabólicos, especialmente nas mulheres; considerando-se a gordura subcutânea, verificou-se forte correlação com a circunferência da cintura (CC) $(r=0,87$ e $r=0,88)$ e com 0 índice de massa corporal (IMC) $(r=0,88$ e $r=0,83)$ em mulheres e homens, respectivamente. ${ }^{3}$

Diferentes medidas antropométricas são utilizadas na prática clínica e em estudos epidemiológicos para se avaliar a adiposidade corporal, por sua praticidade e baixo custo, como a CC, a IMC e a razão cintura/ estatura (RCE). Apontamos, no parágrafo seguinte, algumas características dessas medidas.

\section{O uso dessas medidas na população idosa demanda a definição de pontos de corte $(P C)$ que considerem as mudanças na composição corporal decorrentes do envelhecimento. Diversos critérios são utilizados para classificar o estado nutricional de idosos a partir do IMC, não havendo consenso na literatura quanto aos $P$ C adotados.}

O IMC não diferencia massa magra de massa gorda, nem avalia a distribuição da gordura corporal; além disso, a perda de tecido muscular afeta sua validade como indicador de adiposidade em idosos, independentemente de mudanças no peso. ${ }^{4,5} \mathrm{~A}$ CC representa 0 acúmulo de gordura abdominal, sendo fortemente correlacionada com o tecido adiposo visceral, avaliado por métodos de imagem, a exemplo da tomografia computadorizada. ${ }^{4,5} \mathrm{~A}$ RCE foi proposta como indicador de adiposidade central mais robusto do que a CC, por resultar em estimativa não enviesada pela estatura. ${ }^{6}$

0 uso dessas medidas na população idosa demanda a definição de pontos de corte (PC) que considerem as mudanças na composição corporal decorrentes do envelhecimento. Diversos critérios são utilizados para classificar o estado nutricional de idosos a partir do IMC, não havendo consenso na literatura quanto aos PC adotados. ${ }^{7-10}$ Quanto à CC, o Ministério da Saúde não apresenta PC específicos para idosos, somente para adultos em geral. ${ }^{8}$ Em relação à RCE, em 2017 foi proposto um PC como marcador de excesso de peso em idosos brasileiros (idade $\geq 60$ anos), usando-se duas classificações de IMC como referência antropométrica. ${ }^{11}$ Utilizando informações de uma coorte iniciada em 2008, pesquisadores propuseram PC de CC para o diagnóstico de obesidade em idosos ( $\geq 60$ anos) usuários do Sistema Único de Saúde (SUS), de Goiânia/G0. ${ }^{12}$ Apesar da relevância, são raros os estudos na literatura que definiram PC específicos para idosos para relacionar com o excesso de peso.

Considerando-se as mudanças na composição corporal, o objetivo deste estudo foi identificar pontos de corte para CC e RCE com melhor sensibilidade, especificidade e acurácia para discriminar idosos com excesso de peso por sexo, utilizando três critérios de índice de massa corporal como referência antropométrica.

\section{Métodos}

Estudo transversal multicêntrico sobre Fragilidade em Idosos Brasileiros (Fibra, 2008/2009). 0 estudo Fibra foi conduzido com idosos da comunidade, residentes nas áreas urbanas de sete cidades brasileiras, a saber: Campinas/SP, Belém/PA, Poços de Caldas/MG, Ermelino Matarazzo/SP, Campina Grande/PB, Parnaíba/PI e Ivoti/RS.

As cidades foram selecionadas por conveniência. Em cada cidade, as amostras foram obtidas por meio de conglomerados e em dois estágios: setor censitário e domicílio. Os critérios de elegibilidade foram: ter idade $\geq 65$ anos; ser morador permanente no domicílio/ setor censitário; e não ter comprometimentos graves de cognição, saúde física, comunicação e mobilidade (como os acamados e com sequelas graves de acidente vascular encefálico). A descrição e demais informações sobre amostragem, as cotas estimadas e obtidas por idade e sexo em cada cidade e a realização do campo da pesquisa encontram-se publicadas. ${ }^{13}$

As variáveis de interesse foram o sexo (masculino; feminino) e as medidas de peso, estatura e CC, aferidas conforme protocolos clássicos. ${ }^{14}$

Para a aferição do peso, estando com roupas leves, sem objetos nos bolsos, descalço, 0 idoso era posicionado em pé sobre a plataforma da balança, de frente para a 
escala, ereto, com o olhar fixo para a frente, com os pés paralelos e ligeiramente afastados. Para a estatura, 0 idoso deveria estar descalço, posicionado em pé, ereto, de costas para a escala, com os pés unidos e com a cabeça mantida no Plano de Frankfurt.

0 peso e a estatura foram usados para calcular o IMC [peso (kg)/altura ${ }^{2}(\mathrm{~m})$ ]. Para a avaliação do excesso de peso (sobrepeso/obesidade), os valores de IMC foram dicotomizados segundo os PC dos critérios: NSI (IMC $>27$ $\left.\mathrm{kg} / \mathrm{m}^{2}\right),{ }^{7}$ preconizado pelo Ministério da Saúde/Sisvan; ${ }^{8}$ OPAS (IMC $\left.\geq 28 \mathrm{~kg} / \mathrm{m}^{2}\right) ;{ }^{9}$ e OMS (IMC $\left.\geq 25 \mathrm{~kg} / \mathrm{m}^{2}\right) .{ }^{10}$

Para aferir a CC, o avaliado deveria estar em pé. Posicionado de frente para 0 idoso, 0 entrevistador passava a fita métrica em volta do corpo do participante, no ponto médio entre a borda inferior da última costela e a crista ilíaca. ${ }^{14}$

A RCE foi determinada pela divisão do valor da CC pela estatura, em centímetros. Uma revisão sistemática detectou PC de 0,5 para RCE como marcador de risco cardiometabólico para adultos em geral. ${ }^{15} 0$ presente estudo se propôs a estabelecer PC de RCE específicos para idosos de ambos os sexos, considerando-se as alterações que ocorrem na composição corporal por fatores fisiológicos, metabólicos, socioeconômicos e psicológicos. ${ }^{16,17}$

Para as análises, foram utilizados gráficos de curvas ROC para definir os PC para CC e RCE correspondentes à condição de excesso de peso, segundo três critérios de classificação do IMC. A sensibilidade (proporção de teste diagnóstico positivo entre os indivíduos que apresentaram a condição de interesse), a especificidade (proporção de teste diagnóstico negativo entre os indivíduos que não apresentaram a condição) e a acurácia (a proporção de acertos de um teste diagnóstico) foram obtidas utilizando-se o software Stata. Considerou-se como condição de interesse o sobrepeso/obesidade. Também foram feitas comparações de proporções por meio do teste do qui-quadrado de Pearson, considerando-se nível de significância de 5\% e calculados intervalos de confiança de $95 \%\left(\mathrm{IC}_{95 \%}\right)$. As análises estatísticas foram feitas com uso do programa Stata, versão 14.0.

o Estudo Fibra foi aprovado pelo Comitê de Ética em Pesquisa da Faculdade de Ciências Médicas da Universidade Estadual de Campinas em 22 de maio de 2007 e registrado na Plataforma Brasil (Parecer: 208/2007; Certificado de Apresentação para Apreciação Ética CAAE: 39547014.0.1001.5404). Os idosos assinaram o Termo de Consentimento Livre e Esclarecido.

\section{Resultados}

0s dados referem-se a 3.477 idosos, dos quais $67,7 \%$ eram mulheres $(n=2.353)$. As idades variaram entre 65 e 97 anos com média de 73,3 anos nos idosos, e entre 65 e 101 anos com média de 72,8 anos nas idosas. Os homens tiveram média de IMC de $26,2 \mathrm{~kg} /$ $\mathrm{m}^{2}\left(\mathrm{IC}_{95 \%} 26,0-26,5\right)$, e as mulheres, de $27,4 \mathrm{~kg} / \mathrm{m}^{2}$ $\left(\mathrm{IC}_{95 \%} 27,2-27,6\right)$. Em relação à proporção de excesso de peso, observou-se diferença significativa entre os sexos: as proporções entre as mulheres foram de $51,4 \%$, $42,9 \%$ e $67,7 \%$, e entre os homens, de $40,0 \%, 31,0 \%$ e 60,3\%, de acordo com os critérios NSI, OPAS e OMS, respectivamente $(\mathrm{p}<0,001)$.

Os PC obtidos para CC, utilizando-se os critérios da OMS, NSI e OPAS, foram iguais a $86,5 \mathrm{~cm}, 88,7 \mathrm{~cm}$ e $91,5 \mathrm{~cm}$ nas mulheres, e iguais a $93,0 \mathrm{~cm}, 96,0 \mathrm{~cm}$ e $97,5 \mathrm{~cm}$ nos homens. Para ambos os sexos, o critério da NSI resultou em estimativas pontuais mais elevadas para a sensibilidade, os quais corresponderam aos $\mathrm{PC}$ de $88,7 \mathrm{~cm}$ para as idosas e de $96,0 \mathrm{~cm}$ para os idosos (Tabela 1), sem diferença estatisticamente significativa, com sobreposição dos IC $\mathrm{I}_{95 \%}$. Quanto à RCE, os PC foram de 0,57, 0,58 e 0,59 no sexo feminino, e 0,56, 0,58 e 0,59 no sexo masculino, considerando-se os critérios OMS, NSI e OPAS. Nas mulheres, a especificidade e 0 valor preditivo positivo (VPP) foram maiores pela classificação da OMS. Nos homens, o critério da OPAS apresentou maior sensibilidade, e o da OMS, maior VPP (Tabela 1).

Embora expressivos para ambos os sexos, os valores da área sob a curva (acurácia global) não são estatisticamente diferentes, com sobreposição dos IC $_{95 \%}$ (Tabela 1, Figura 1). Para a RCE, a acurácia global foi de 0,87 para os três critérios de classificação de IMC nas mulheres e, entre os homens, variou de 0,90 (OMS) a 0,92 (OPAS). Por sua vez, para a CC, os valores de acurácia global foram de 0,87 nas mulheres e em torno de 0,90 nos homens. Em ambos os sexos, não foram detectadas diferenças significativas para os resultados de área sob a curva, dada a sobreposição dos IC ${ }_{95 \%}$ (Tabela 1, Figura 2).

\section{Discussão}

Os principais achados foram: verificar PC de CC $\mathrm{e}$ RCE para o diagnóstico do excesso de peso em idosos segundo três critérios de classificação do IMC; os valores de corte obtidos com os critérios da NSI e da OPAS 
Tabela 1 - Sensibilidade e especificidade dos pontos de corte para a circunferência da cintura (CC) e razão cintura/estatura (RCE) em relação a três critérios padrão ouro de índice de massa corporal (IMC) em idosos, de ambos os sexos, Estudo Fibra, 2008/2009

\begin{tabular}{|c|c|c|c|c|c|}
\hline Sexo & $\begin{array}{l}\text { Ponto de } \\
\text { corte }\end{array}$ & $\begin{array}{c}\text { Sensibilidade } \\
(\%)\end{array}$ & $\begin{array}{c}\text { Especificidade } \\
(\%)\end{array}$ & Área sob a curva $\left(\mathrm{IC}_{95 \%}\right)$ & $\begin{array}{c}\text { Valor preditivo positivo } \\
(\%)\end{array}$ \\
\hline & \multicolumn{5}{|c|}{ Circunferência da cintura (CC) } \\
\hline \multirow{3}{*}{ Feminino } & $\geq 86,5^{\mathrm{a}}$ & 79,39 & 79,02 & $\begin{array}{c}0,87 \\
(0,86 ; 0,89)\end{array}$ & 88,82 \\
\hline & $\geq 88,7^{b}$ & 83,00 & 76,69 & $\begin{array}{c}0,87 \\
(0,85 ; 0,88)\end{array}$ & 79,03 \\
\hline & $\geq 91,5^{c}$ & 75,71 & 82,12 & $\begin{array}{c}0,87 \\
(0,85 ; 0,88)\end{array}$ & 76,10 \\
\hline \multirow{4}{*}{ Masculino } & $\geq 93,0^{\mathrm{a}}$ & 80,42 & 82,39 & $\begin{array}{c}0,89 \\
(0,87 ; 0,91)\end{array}$ & 87,41 \\
\hline & $\geq 96,0^{b}$ & 83,72 & 83,16 & $\begin{array}{c}0,90 \\
(0,88 ; 0,92)\end{array}$ & 76,85 \\
\hline & $\geq 97,5^{c}$ & 81,55 & 84,05 & $\begin{array}{c}0,90 \\
(0,88 ; 0,92)\end{array}$ & 69,72 \\
\hline & \multicolumn{5}{|c|}{ Razão cintura/estatura (RCE) } \\
\hline \multirow{3}{*}{ Feminino } & $\geq 0,57^{a}$ & 79,03 & 81,54 & $\begin{array}{c}0,87 \\
(0,85 ; 0,88)\end{array}$ & 89,99 \\
\hline & $\geq 0,58^{b}$ & 80,42 & 78,28 & $\begin{array}{c}0,87 \\
(0,86 ; 0,89)\end{array}$ & 79,67 \\
\hline & $\geq 0,59^{c}$ & 79,49 & 79,06 & $\begin{array}{c}0,87 \\
(0,86 ; 0,87)\end{array}$ & 74,06 \\
\hline \multirow{3}{*}{ Masculino } & $\geq 0,56^{\mathrm{a}}$ & 82,63 & 81,94 & $\begin{array}{c}0,90 \\
(0,88 ; 0,92)\end{array}$ & 87,43 \\
\hline & $\geq 0,58^{b}$ & 82,26 & 85,84 & $\begin{array}{c}0,91 \\
(0,89 ; 0,93)\end{array}$ & 79,50 \\
\hline & $\geq 0,59^{c}$ & 88,02 & 83,66 & $\begin{array}{c}0,92 \\
(0,91 ; 0,94)\end{array}$ & 70,81 \\
\hline
\end{tabular}

a) Ponto de corte considerando a categoria de sobrepeso/obesidade proposto pela $0 M S$ (IMC $\geq 25 \mathrm{Kg} / \mathrm{m}^{2}$ ). b) Ponto de corte considerando a categoria de sobrepeso proposto pela NSI/Lipschitz (IMC $\left.>27 \mathrm{Kg} / \mathrm{m}^{2}\right)$.

c) Ponto de corte considerando a categoria de sobrepeso/obesidade proposto pela OPAS (IMC $\left.\geq 28 \mathrm{Kg} / \mathrm{m}^{2}\right)$.

mostraram-se maiores comparados ao da OMS, mas sem diferenças significativas.

0 excesso de peso foi superior nas mulheres, em todas as referências de IMC, resultado amplamente descrito na literatura, ${ }^{18-20}$ explicado por diferenças fisiológicas que determinam 0 acúmulo e a redistribuição de gordura no envelhecimento. ${ }^{1,2}$ Para ambos os sexos, os critérios de IMC produziram valores de CC e RCE similares. Os PC detectados para CC foram superiores aos estabelecidos pela OMS para a população adulta (homens $\geq 94 \mathrm{~cm}$; mulheres $\geq 80 \mathrm{~cm}) \cdot{ }^{10}$ Analisando uma amostra de idosos usuários do SUS de Goiânia/G0, com idade $\geq 60$ anos, Silveira et al. ${ }^{12}$ propuseram PC de CC de $98,8 \mathrm{~cm}$ no sexo masculino e $90,5 \mathrm{~cm}$ no sexo feminino. Os PC obtidos neste estudo ficaram entre $6,5 \mathrm{~cm}$ e $11,5 \mathrm{~cm}$ superiores ao recomendado pela OMS para mulheres, e entre menos $1 \mathrm{~cm}$ e mais $3,5 \mathrm{~cm}$ do $\mathrm{PC}$ estabelecido para homens adultos, em geral. Em relação ao $\mathrm{PC}$ proposto por Silveira et al., ${ }^{12}$ este estudo identificou diferenças em torno de
$1 \mathrm{~cm}$ para homens e mulheres, considerando-se o PC de CC resultante do critério da OPAS.

Quanto à RCE, foram identificados PC que se aproximaram do 0,55 encontrado por Corrêa et al. ${ }^{11} \mathrm{em}$ 2008/2009, em uma amostra representativa da população brasileira ( $\geq 60$ anos), com características sociodemográficas semelhantes àquelas dos idosos incluídos nesta pesquisa. 0 estudo de Corrêa et al. ${ }^{11}$ foi o primeiro no Brasil a detectar o PC da RCE para identificação do excesso de peso em idosos, utilizando os critérios de classificação do IMC da NSI e OMS como referência antropométrica. 0 presente estudo agrega informações à literatura e pode contribuir para ampliar a discussão científica sobre o uso dessas medidas antropométricas em idosos.

Comparada ao IMC, a CC é um preditor mais robusto de mortalidade e risco de doença cardiovascular., ${ }^{2,411,21}$ Dados de coortes prospectivas detectaram maior incidência de doença cardíaca em indivíduos com excesso de 


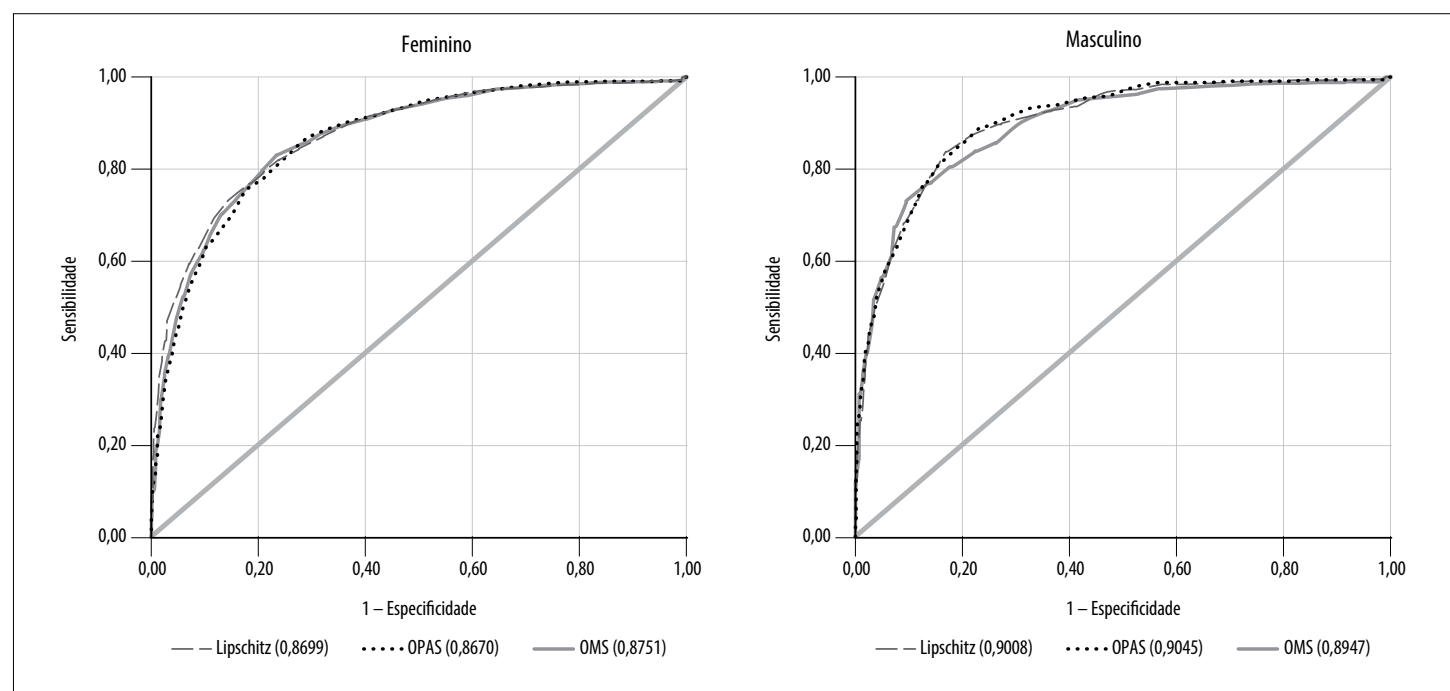

Figura 1 - Curvas receiver operating characteristic (ROC) da circunferência da cintura (CC), segundo o sexo e três critérios padrão ouro de índice de massa corporal (IMC), Estudo Fibra, 2008/2009
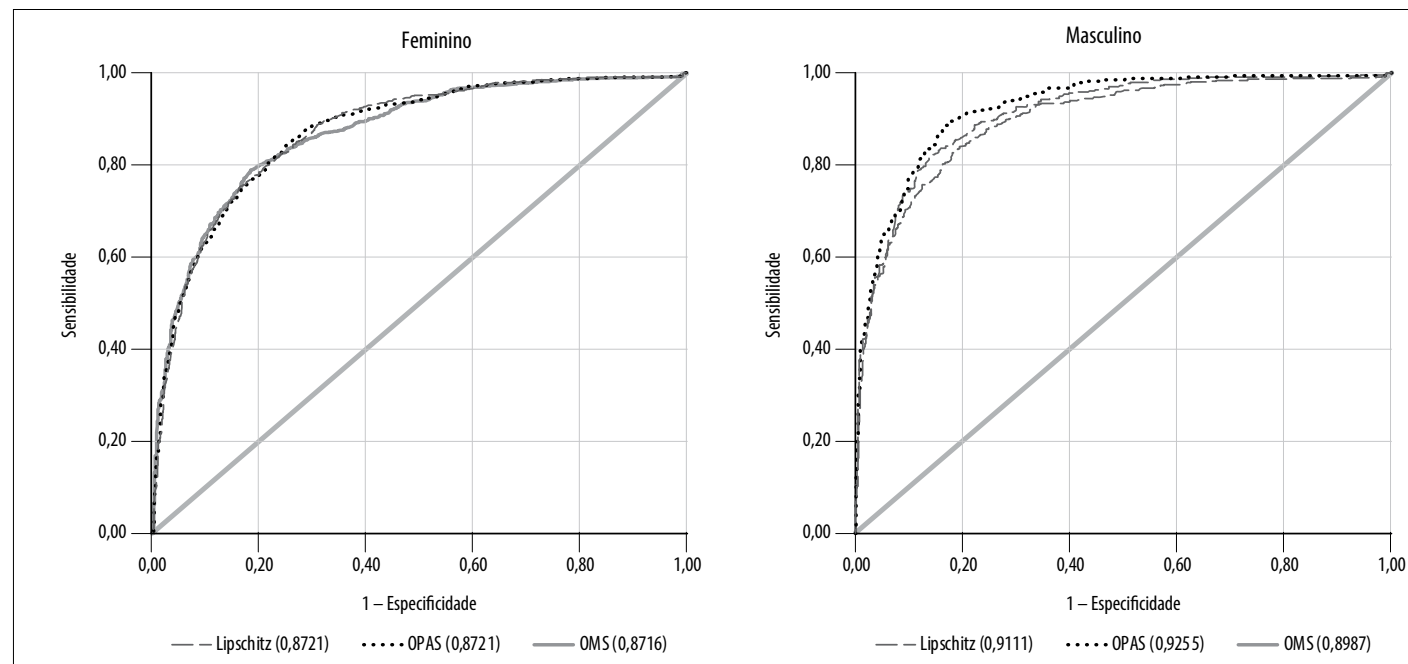

Figura 2 - Curvas receiver operating characteristic (ROC) da razão cintura/estatura (RCE), segundo o sexo e três critérios padrão ouro de índice de massa corporal (IMC), Estudo Fibra, 2008/2009

peso (IMC $\left.\geq 25 \mathrm{~kg} / \mathrm{m}^{2}\right)$ e com CC aumentada $(\geq 94 \mathrm{~cm}$; $\geq 80 \mathrm{~cm}),{ }^{4}$ e maior risco de mortalidade geral e por causas específicas com 0 aumento da CC e do IMC. ${ }^{21}$ 0 uso da RCE tem sido recomendado em substituição às medidas de CC e IMC (isoladas ou combinadas), por apresentar superioridade na predição de fatores de risco cardiometabólicos., ${ }^{5,6,11}$

Como limitação, deve-se considerar um possível viés de seleção. 0 Estudo Fibra fez opção por idosos que compareceram aos locais de coleta de dados por seus próprios meios, tornando provável a participação majoritária do estrato com maior competência física, excluindo os idosos acamados e com sequelas graves de acidente vascular encefálico.

Conclui-se que, independentemente do critério de IMC usado para a definição de excesso de peso, os PC para CC e RCE foram similares. Na avaliação antropométrica dos idosos, devem-se considerar PC mais adequados ao seu perfil nutricional. Avanços neste campo da ciência podem contribuir para o melhor diagnóstico de 
doenças crônicas relacionadas à obesidade no âmbito populacional e ambulatorial. Entre as implicações para os serviços de saúde, destaca-se a aplicação de PC mais acurados para o diagnóstico e a vigilância de idosos com risco cardiometabólico.

\section{Contribuições das autoras}

Assumpção D e Francisco PMSB participaram da concepção e delineamento do artigo, análise e interpretação dos dados, elaboração do manuscrito e revisão

\section{Referências}

1. Beaufrere B, Morio B. Fat and protein redistribution with aging: metabolic considerations. Eur J Clin Nutr [Internet]. 2000 Jun [cited 2020 May 22];54 Suppl 3:\$48-53. Available from: https://doi.org/10.1038/ sj.ejcn. 1601025

2. Scafoglieri A, Provyn S, Bautmans I, Van Roy P, Clarys JP. Direct relationship of body mass index and waist circumference with body tissue distribution in elderly persons. J Nutr Health Aging [Internet]. 2011 Dec [cited 2020 May 22];15(10):924-31. Available from: https://doi.org/10.1007/s12603-010-0272-x

3. Fox CS, Massaro JM, Hoffmann U, Pou KM, MaurovichHorvat P, Liu C-Y, et al. Abdominal visceral and subcutaneous adipose tissue compartments: association with metabolic risk factors in the framingham heart study. Circulation [Internet]. 2007 Jul [cited 2020 May 22];116(1):39-48. Available from: https://doi.org/10.1161/circulationaha.106.675355

4. Flint AJ, Rexrode KM, Hu FB, Glynn RJ, Caspard $\mathrm{H}$, Manson JE, et al. Body mass index, waist circumference, and risk of coronary heart disease: a prospective study among men and women. Obes Res Clin Pract [Internet]. 2010 Jul [cited 2020 May 22];4(3):e171-e181. Available from: https://dx.doi. org/10.1016\%2Fj.orcp.2010.01.001

5. Associação Brasileira para o Estudo da Obesidade e da Síndrome Metabólica - ABESO. Diretrizes brasileiras de obesidade 2016 [Internet]. 4. ed. São Paulo: Associação Brasileira para o Estudo da Obesidade e da Síndrome Metabólica; 2016 [citado 2019 mar 5]. 186 p. Disponível em: https://portaldeboaspraticas. iff.fiocruz.br/biblioteca/diretrizes-brasileiras-deobesidade-2016-abeso/ crítica do conteúdo intelectual. Ferraz Ro desenvolveu as análises estatísticas, participou da concepção e delineamento do estudo e da revisão crítica do conteúdo do manuscrito. Borim FSA participou da concepção e delineamento do estudo e da revisão crítica do conteúdo intelectual. Neri AL realizou a concepção do Estudo Fibra, participou da concepção e delineamento do estudo e da revisão crítica do conteúdo intelectual. Todas as autoras contribuíram na redação, aprovaram a versão final e assumem responsabilidade por todos os aspectos do manuscrito, garantindo a integridade da obra.
6. Schneider HJ, Klotsche J, Silber S, Stalla GK, Wittchen $\mathrm{H}-\mathrm{U}$. Measuring abdominal obesity: effects of height on distribution of cardiometabolic risk factors risk using waist circumference and waist-to-height ratio. Diabetes Care [Internet]. 2011 Jan [cited 2020 May 22];34(1):e7. Available from: https://doi.org/10.2337/ dc10-1794

7. Cervi A, Franceschini SCC, Priore SE. Análise crítica do uso do índice de massa corporal para idosos. Rev Nutr [Internet]. 2005 nov-dez [citado 2020 maio 22];18(6):765-75. Disponível em: http://dx.doi. org/10.1590/S1415-52732005000600007

8. Ministério da Saúde (BR). Orientações para coleta e análise de dados antropométricos em serviços de saúde: norma técnica do sistema de Vigilância Alimentar e Nutricional - SISVAN [Internet]. Brasília: Ministério da Saúde; 2011 [citado 2019 fev 12]. 76 p. Disponivel em: http://dab.saude.gov.br/portaldab/ biblioteca.php?conteudo $=$ publicacoes $/$ orientacoes coleta_analise_dados_antropometricos

9. Organización Panamericana de la Salud. XXXVI Reunión del Comité Asesor de Investigaciones en Salud. Encuesta Multicéntrica: Salud Bienestar y Envejecimiento (SABE) en América Latina y el Caribe [Internet]. Washington, D.C: Organización Panamericana de la Salud; 2001 [citado 2019 fev 15]. 93 p. Disponível em: http://envejecimiento.csic.es/ documentos/documentos/paho-salud-01.pdf

10. World Health Organization - WHO. Obesity: preventing and managing the global epidemic [Internet]. Geneva: World Health Organization; 2000 [cited 2019 Fev 12]. 252 p. Available from: https:/www.who.int/nutrition/ publications/obesity/WHO_TRS_894/en/ 
11. Corrêa MM, Tomasi E, Thumé E, Oliveira ERA, Facchini LA. Razão cintura-estatura como marcador antropométrico de excesso de peso em idosos brasileiros. Cad Saúde Pública [Internet]. 2017 [citado 2020 maio 22];33(5):e00195315. Disponível em: http://dx.doi.org/10.1590/0102-311x00195315

12. Silveira EA, Pagotto V, Barbosa LS, Oliveira C, Pena GG, Velasquez-Melendez G. Acurácia de pontos de corte de IMC e circunferência da cintura para a predição de obesidade em idosos. Ciên Saúde Coletiva [Internet]. 2020 mar [citado 2020 maio 22];25(3):1073-82. Disponível em: http://dx.doi.org/10.1590/141381232020253.13762018

13. Neri AL, Yassuda MS, Araújo LF, Eulálio MC, Cabral $\mathrm{BE}$, Siqueira MEC, et al. Metodologia e perfil sociodemográfico, cognitivo e de fragilidade de idosos comunitários de sete cidades brasileiras: Estudo FIBRA. Cad Saúde Pública [Internet]. 2013 abr [citado 2020 maio 22];29(4):778-92. Disponível em: http:// dx.doi.org/10.1590/S0102-311X2013000400015

14. World Health Organization. Physical status: the use and interpretation of anthropometry [Internet]. Geneva: World Health Organization; 2003 [cited 2019 Feb 12]. 452 p. Available from: https://www.who.int/ childgrowth/publications/physical_status/en/

15. Browning LM, Hsieh SD, Ashwell M. A systematic review of waist-to-height ratio as a screening tool for the prediction of cardiovascular disease and diabetes: 0.5 could be a suitable global boundary value. Nutr Res Rev [Internet]. 2010 Dec [cited 2020 May 22];23(2):247-269. Available from: https://doi. org/10.1017/s0954422410000144
16. Amarya S, Singh K, Sabharwal M. Changes during aging and their association with malnutrition. J Clin Gerontol Geriatr [Internet]. 2015 Sep [cited 2020 May 22];6(3):78-84. Available from: https://doi. org/10.1016/j.jcgg.2015.05.003

17. Ahmed T, Haboubi N. Assessment and management of nutrition in older people and its importance to health. Clin Interv Aging [Internet]. 2010 Aug [cited 2020 May 22];9(5):207-16. Available from: https://doi. org/10.2147/cia.s 9664

18. Pereira IFS, Spyrides MHC, Andrade LMB. Estado nutricional de idosos no Brasil: uma abordagem multinível. Cad Saúde Pública [Internet]. 2016 maio [citado 2020 maio 22];32(5):e00178814. Disponível em: http://dx.doi.org/10.1590/0102-311X00178814

19. Rezende FAC, Ribeiro AQ, Priore SE, Franceschinni SCC. Anthropometric differences related to genders and age in the elderly. Nutr Hosp [Internet]. 2015 Aug [cited 2020 May 22];32(2):757-64. Available from: https://doi.org/10.3305/nh.2015.32.2.8641

20. Lima-Costa MF, Firmo JOA, Uchôa E. The Bambuí cohort study of aging: methodology and health profile of participants at baseline. Cad Saúde Pública [Internet]. 2011 [cited 2020 May 22];27 Suppl 3:32735. Available from: http://dx.doi.org/10.1590/S0102311X2011001500002

21. Leitzmann MF, Moore SC, Koster A, Harris TB, Park Y, Hollenbeck A, et al. Waist circumference as compared with body-mass index in predicting mortality from specific causes. PLoS One [Internet]. $2011 \mathrm{Apr}$ [cited 2020 May 22];6(4):e18582. Available from: https:// doi.org/10.1371/journal.pone.0018582 


\begin{abstract}
Objective: to identify cut-off points for waist circumference (WC) and waist-to-beight ratio (WHtR) with better sensitivity, specificity and accuracy to distinguish overweight elderly people by sex, using three body mass index criteria as anthropometric references. Methods: this was a cross-sectional study that analyzed weight, height and WC measurements of 3,477 elderly people (20082009); receiver operating characteristic (ROC) curves were used to define the cut-off points. Results: WC cut-off points were $86.5 \mathrm{~cm}, 88.7 \mathrm{~cm}$ and $91.5 \mathrm{~cm}$ in women, and $93.0 \mathrm{~cm}, 96.0 \mathrm{~cm}$ and $97.5 \mathrm{~cm}$ in men, according to World Health Organization/WHO, Nutrition Screening Initiative/ NSI and Pan American Health Organization/PAHO criteria, respectively; WHtR cut-off points corresponded to 0.57, 0.58, 0.59 in the female sex, and 0.56, 0.58 and 0.59 in the male sex; highest absolute overall accuracy values: PAHO (men) and WHO (women). Conclusion: WC and WHtR cut-off points were similar, regardless of the BMI criterion adopted.

Keywords: Aged; Waist Circumference; Body Mass
\end{abstract} Index; Waist-Height Ratio; Health Surveys; Cross-Sectional Studies.

\section{Resumen}

objetivo: identificar puntos de corte para circunferencia de cintura (CC) y razón cintura-estatura (RCE) con mejor sensibilidad, especificidad y precisión, discriminando adultos mayores con exceso de peso por sexo, utilizando tres criterios de indice de masa corporal como referencia antropométrica. Métodos: estudio transversal que analizó peso, estatura y CC de 3.477 adultos mayores (2008-2009). Se utilizaron gráficos de curvas ROC (receiver operating characteristic) para definir puntos de corte. Resultados: los puntos de cortes para CC fueron de $86,5 \mathrm{~cm}, 88,7 \mathrm{~cm}$ y $91,5 \mathrm{~cm}$ para mujeres, y de 93,0 cm, 96,0 cm y 97,5 cm para bombres, por criterios de la Organización Mundial de la Salud/ OMS, Nutrition Screening Initiative/NSI y Organización Panamericana de la Salud/OPS, respectivamente. Para la RCE 0,57, 0,58, 0,59 correspondieron al sexo femenino, y 0,56, 0,58 y 0,59 al masculino; mayores valores absolutos de exactitud global: OPS (hombres) $y$ OMS (mujeres). Conclusión: los puntos de corte de CC $y$ RCE fueron similares, independientemente del criterio de IMC adoptado.

Palabras clave: Anciano; Circunferencia de la Cintura; Índice de Masa Corporal; Relación Cintura-Estatura; Encuestas Epidemiológicas; Estudios Transversales.

Recebido em 14/12/2019

Aprovado em 13/05/2020 


\section{Errata}

No artigo "Pontos de corte da circunferência da cintura e da razão cintura/estatura para excesso de peso: estudo transversal com idosos de sete cidades brasileiras, 2008-2009", com número de doi: 10.5123/\$1679-49742020000300003, publicado no periódico Epidemiologia e Serviços de Saúde, 29(4):e2019502, 2020, na página 01:

Onde se lia:

doi: $10.5123 / \$ 1679-49742020000300003$

Leia-se:

doi: $10.5123 / \mathrm{S} 1679-49742020000400027$ 